\title{
Shoulder Pain in Swimmers
}

\section{Gomaa M. Othman}

Dept. Of Theories and Applications of Aquatic sports, Faculty of Physical Education, Zagazig university, zagazig, Egypt

Corresponding Author: Gomaa M. Othman, Dept. Of Theories and Applications of Aquatic sports, Faculty of Physical Education, Zagazig university,zagazig, Egypt E-mail: Dr.gomaaothman@yahoo.com.

Received date: November 11, 2019; Accepted date: December 04, 2019; published date: December 09, 2019

Citation: Gomaa M. Othman, (2019) Shoulder Pain in Swimmers.J. Orthopaedics and Surgical Sports Medicine, 2(1): Doi:10.31579/26410427/018.

Copyright: (c) 2019 Gomaa M. Othman. This is an open-access article distributed under the terms of The Creative Commons Attribution License, which permits unrestricted use, distribution, and reproduction in any medium, provided the original author and source are credited.

\begin{abstract}
Shoulder pain is the most important symptom that affects competitive swimmers, with a prevalence between $40-91 \%$ [1-2] , and it constitutes a special syndrome called the "swimmer's shoulder".

This syndrome, described by Kennedy and Hawkins in 1974 [2] consists in discomfort after swimming activities in a first step. This may progress to pain during and after training. Finally, the pain affects the pro23wsq2wgress of the athlete [3].
\end{abstract}

Keywords: pain; biomechanics; shoulder

\section{Introduction}

Shoulder pain is the most important symptom that affects competitive swimmers, with a prevalence between $40-91 \%$ [1-2], and it constitutes a special syndrome called the "swimmer's shoulder".

This syndrome, described by Kennedy and Hawkins in 1974 [2] consists in discomfort after swimming activities in a first step. This may progress to pain during and after training. Finally, the pain affects the progress of the athlete [3]. Some researchers have demonstrated that an important proportion of competitive swimmers have shoulder pain that interferes with training and progress of their abilities. The percentage of athletes with swimmer's shoulder is proportional to the age, the years of practice and the level of competition. Swimmers with interfering pain might not progress in training and thus will not compete as effectively [4].

One of the first reports of this problem was in the 1972 Olympic Games in Munich; Kennedy noticed a high incidence of shoulder pain among swimmers of Canadian group: of 35 competitive swimmers, there were 43 orthopaedic consultations, with 16 specific-related to shoulder (37\%), being the most frequent problem [3].

Kennedy had performed a cross-Canada survey involving all competitive swimmers (5000 yards per day). A total of 2496 swimmers were included, reporting a $3 \%$ (81 swimmers) shoulder complaints, caused primarily by the freestyle and butterfly strokes and occasionally by the backstroke [3].

\section{Shoulder Biomechanics in Swimming}

Swimming requires several different shoulder motions, most being performed during circumduction in clockwise and counter-clockwise directions with varying degrees of internal and external rotation and scapular protraction and retraction [5].

Competitive swimmers used four types of strokes: freestyle or front crawl stroke, breaststroke, backstroke, and butterfly stroke. The fastest, most popular and most widely used stroke for training is the freestyle stroke [6]. The power for this stroke comes $80 \%$ from the pull and $20 \%$ from the kick [6].

\section{The freestyle stroke pull-cycle can be divided in four} phases [7]:

1. Early pull-through: beginning with the hand entry into the water and ending when the humerus is perpendicular to the axis of the torso.

2. Late pull-through: beginning at the completion of early pullthrough and ending as the hand leaves the water.

3. Early recovery: beginning at hand exit and ending when the humerus is perpendicular to the water surface.

4. Late recovery: beginning at the completion of early recovery and ending at hand entry.

During the entry and beginning of the pull phases, the glenohumeral joint is in forward flexion, and the humerus is in abduction and internal rotation [6]. During the end of the pull, the joint is extended and the humerus is in adduction and internal rotation [6]. During the recovery period, the arm is in abduction and internal rotation, moving from extension to flexion above the water [6].

The backstroke is considered the complement to the freestyle stroke, and the arm actions involve the same four phases; however, power comes $25 \%$ from the kick and $75 \%$ from the pull [6].

The butterfly stroke is performed with the arms in the same phase of the stroke at one time. During the entry, both shoulders are flexed, abducted, and internally rotated. During the pull-through phase, the shoulders move into extension, and in the recovery, the arms are brought above the water from extension to flexion while abducted and internally rotated. The power for this stroke comes $30 \%$ from the kick and $70 \%$ from the pull [6].

The breaststroke has a fifty-fifty split from where the power is initiated. In the pull phase, the arms move into adduction, internally rotated, and are always below the water surface. During the recovery, the arms return in a circular pattern, always under the water surface [6]. In 1991 Marilyn Pink performed the most detailed electromyographic and cinematographic analysis of freestyle stroke [7]. In the pull-through phase, they recognized three different phases: the first phase was 
reaching forward and gliding. From the point that the hand entered the water to the point of maximal elbow extension, there was no actual pulling. Pulling began after the reach.

Reach began as the hand entered the water (predominance of phasic activity in the upper trapezius, rhomboids, supraspinatus, and the anterior and middle deltoids). The serratus anterior was upwardly rotating and protracting the scapula while the upper trapezius was elevating it and the rhomboids were retracting it.

Therefore, the hand followed an S-shaped curve during the pull-through phase (pectoralis major is the responsible for the initial powerful adduction and extension of the humerus). When the humerus is perpendicular to the body, latissimus dorsi continued the pulling by shoulder extension (internal rotation is given by subscapularis). Also, the serratus anterior was acting to move the body over the arm and through the water and upwardly rotate the scapula to maintain glenohumeral joint congruency. When the latissimus dorsi finished its activity, the posterior deltoid fired to lift the shoulder out of the water.

Finally, in the recovery position (much shorter), the activity noted at the end of pull-through in the middle deltoid and supraspinatus is maintained. The rhomboids fired to retract the scapula. Pink et al highlighted that the subscapularis and the serratus anterior continually fire above $20 \%$ MMT (manual muscle test). Thus, these two muscles would appear to be susceptible to fatigue [7].

\section{Etiology}

The term "swimmer's shoulder" covers a spectrum of consecutive or coexisting pathologies, with rotator cuff-related pain to be the most common finding [8].

Kennedy and Hawkins [3] proposed that the avascularity zones of the supraspinatus and bicipital tendon in the adducted position of the arm are the explanation of swimmer's shoulder. When the shoulders are abducted, all of the vessels of the tendons are almost completely filled. However, when the arm is at the side in the adducted position, there is a constant area of avascularity extending $1 \mathrm{~cm}$. proximal directly to the point of insertion of the supraspinatus and in the intracapsular portion of the bicipital tendon when it passes over the head of the humerus [3] Bak reported that the main factor in the development of a swimmer's shoulder seems to be the high training volume during growth in the absence of a well-designed and balanced dryland training program, affecting the muscular balance and the scapular motion [8].

A clear consensus is lacking as to the causes of shoulder pain in swimmers. A general medical assumption has been that swimmer's shoulder is a rotator cuff pathology [9]. Kennedy and Hawkins explain this phenomenon based on the differential vascularity of the supraspinatus and bicipital tendons [3]. Other reports suggest that the impingement is produced by glenohumeral instability or muscular imbalance of the scapular stabilizers (secondary impingement). [6,10,11] Indeed, the muscular electric activity is different in the shoulders with pain during the swimming $[8,12]$.

Essentially, there are various causes or contributor factors accepted to cause shoulder pain in swimmers. The intrinsic mechanism has been defined as a tendon injury that originates within the tendon from direct tendon overload, intrinsic degeneration, or other insult. The extrinsic mechanism has been defined as tendon damage caused by injury of the tendon through compression against surrounding structures, specifically the coracoacromial arch. Among these are: overuse, overload, bony configuration, hypovascularity, muscular imbalance, scapular dyskinesis, joint stability, flexibility, stroke technique, training errors, performance level and coaching factors [6].

Brushøj et al [13] in 2007 reported the arthroscopic findings of 18 competitive swimmers. The most common finding at arthroscopy was labral pathology in $11(61 \%)$ shoulders. Of these, five had signs of posterior superior impingement, two in combination with subacromial impingement. The second most common finding was subacromial impingement (28\%). Only two swimmers had isolated inflammation of the bicipital tendon.

\section{Overuse}

The repeated movement of the shoulder can cause micro injury to different structures under risk during swimming. The elite swimmers may $\log$ up to 8000-20000 meters per day average using the freestyle arm stroke for most of the distance [6]. At an average of 8-10 arm cycles per 25 meters, a swimmer completes over one million shoulder rotations each week [14]. Calculated 396000 strokes per season in male competitive swimmers and declared that it is remarkable to them that an even greater number of shoulder problems do not develop.

\section{Impingement and Supraspinatus Tendinopathy}

Swimming involves repetitive overhead movement [15]. Jobe et al [16] hypothesized that repetitive and forceful overhead activity causes a gradual stretching out of the anteroinferior capsuloligamentous structures leading to mild laxity, instability and impingement.

\section{Shoulder instability and range of motion}

The primary stabilizer of the shoulder joint is the capsulolabral complex (static stabilizer). The rotator cuff muscles function dynamically as secondary stabilizers by contracting in a coordinated and synergistic fashion to contain the humeral head throughout abduction. The deltoid functions in a force-couple with the internal rotator and external rotator muscles to maintain the humeral head centered in the glenoid during arm elevation

[17].

Imbalances of the rotator musculature, excess capsular laxity, or loss of capsular flexibility, have all been implicated as etiologic factors in both glenohumeral instability and impingement syndrome

\section{Training Errors}

A rapidly increase in the hours or distance per day is a classic training error. The high level of repetitions can led to fatigue and is the start of the pathological way to swimmer's shoulder, so if the swimmer progression is too aggressive or if he has reached a plateau and some discomfort has appear. A modification of the swim distance may need to be done and/or an increase in the dry-land activities to prevent a progression of an injury.

\section{Treatment Strategies}

The first time the swimmers experience pain, usually complaints in the subacromial region. The symptoms are related to an inflammatory condition (bursitis, tendonitis) and labeled as impingement syndrome. As we have learned, impingement is a consequence of a subtle or evident imbalance in the shoulder that produces an antero/superior migration of the head by imbalance forces or tissues that can be corrected.

According to Bak [8], when the pain is only at swimming (phase 1), the first strategy is to active rest, reduce training and use icepack after training. The coach should look for technical stroke analysis and correction. Exercise directed toward specific dysfunction. The best documentation of scapular stabilizing exercises is for the low rows, lawn mower, robbery, shrugs and push-ups $[18,19]$.

When the pain is daily and not related to swimming practice (phase 2), the strategy is to rest [8]. Swimming should not be allowed for 1 o 2 weeks. A short course of nonesteroidal anti-inflammatory drugs for 5 to 7 days may be prescribed. Injection of corticosteroid in the bursa is not advisable; this practice is at least controversial. Once the pain is tolerable, 
direct exercise can continue. If the pain persist despite of the rest and treatment for more than 3 months [8]. Imaging and a complete study should be done and other strategies should be addressed. Surgical strategies should be considered.

\section{References:}

1. Bak K, Faun $\mathrm{P}$ (1997) Clinical findings in competitive swimmers. Am J Sports Med. 25:254-260.

2. Kennedy JC, Hawkins RJ (1974) Swimmers shoulder. Phys Sports Med. 2:34-38.

3. Kennedy JC, Hawkins R, WB Krissoff (1978) Orthopaedic manifestations of swimming. Am J Sports Med 6(6): 309-322.

4. McMaster WC, Troup J (1993) A survey of interfering shoulder pain in United States competitive swimmers. Am J Sports Med. 21(1):67-70.

5. Tovin BJ (2006) Prevention and Treatment of Swimmer's Shoulder. N Am J Sports PhysTher. 1(4):166-175.

6. King D (1995) Glenohumeral joint impingement in Swimmers. J Athl Train. 30(4):333-337.

7. Pink M, Perry J, Browne A, Scovazzo ML, Kerrigan J (1991) the normal shoulder during freestyle swimming. An electromyographic and cinematographic analysis of twelve muscles. Am J Sports Med. 19(6):569-576.

8. Bak K (2010) The Practical Management of Swimmer's Painful Shoulder: Etiology,Diagnosis, and Treatment. Clin J Sport Med 20:386-390.

9. Hawkins RJ, Kennedy JC (1980) Impingement syndrome in athletes. Am J Sports Med.8:151-158.

10. D Schmitt L, Snyder-Mackler L (1999) Role of scapular stabilizers in etiology and treatment of impingement syndrome. J Orthop Sports Phys Ther. 29:31-38.
11. Su KP, Johnson MP, Gracely EJ, Karduna AR (2004) Scapular Rotation in Swimmers with and without Impingement Syndrome: Practice Effects. Med Sci Sports Exerc. 36:11171123.

12. Scovazzo ML, Browne A, Pink M, Jobe FW, Kerrigan J (1991) The painful shoulder during freestyle swimming. An electromyographic cinematographic analysis of twelve muscles. Am J Sports Med. 19:577-582.

13. Brushj C, Bak K, Johannsen HV, Faun P (2007) Swimmers' painful shoulder arthroscopic findings and return rate to sports. Scand J Med Sci Sports 17:373-377

14. Kammer CS, Young CC, Niedfeldt MW (1999) Swimming injuries and illnesses. Phys Sports Med. 27:51-60.

15. Sein ML, Walton J, Linklater J, Appleyard R, Kirkbride B, Kuah D, Murrell G (2010) Shoulder pain in elite swimmers: primarily due to swim-volume-induced supraspinatus tendinopathy. Br J Sports Med 44:105-113

16. Jobe FW, Kvitne RS, Giangarra CE (1989) Shoulder pain in the overhand or throwing athlete. The relationship of anterior instability and rotator cuff impingement. Ortho Rev 18:963975.

17. Warner JJP, Micheli LJ, Arslanian LE, Kennedy J, Kennedy R (1990) Patterns of

flexibility, laxity, and strength in normal shoulders and shoulders with instability and impingement. Am. J. Sports Med. 18(4):366-375

18. Ludewig PM, Reynolds JF (2009) The association of scapular kinematics and glenohumeral joint pathologies. J Orthop Sports Phys Ther. 39:90-104

19. Kibler WB, Sciascia AD, Uhl TL, Tambay N, Cunningham T (2008) Electromyographic analysis of specific exercises for scapular control in early phases of shoulder rehabilitation. Am J Sports Med. 36:1789-1798. 